\title{
Role of serum urate in neurocognitive function and dementia: new evidence contradicts old thinking
}

\author{
Jasvinder A Singh ${ }^{1,2,3}$
}

In $A R D$, Latourte et al used the data from a community-based prospective French cohort study of healthy 4931 elderly people 65 years or older, examined at six clinical visits (including cognitive examinations) over 12 years, and analysed 1598 participants with a baseline serum urate level (serum uric acid (sUA)), no diagnosis of dementia, a Mini-Mental State Examination (MMSE) score of $>24$ and at least one follow-up visit. ${ }^{1}$ Dementia was diagnosed in a 3 -step process, screening using the MMSE and the Isaacs Set Test by trained psychologists, additional neuropsychological testing by a physician, and adjudication based on criteria by an independent committee of neurologists. Dementia developed in 110 subjects during the 13357 person years of follow-up. Multivariable-adjusted HR with the highest $(\geq 5.8 \mathrm{mg} / \mathrm{dL}$ in men, $\geq 4.9 \mathrm{mg} / \mathrm{dL}$ in women) versus the lowest sUA quartile ( $\leq 4.37$ and $\leq 3.51 \mathrm{mg}$ / $\mathrm{dL}$, respectively) was 1.79 for incident dementia (95\% CI 1.17 to 2.73 ; $p=0.007$ ). A strong association was seen with vascular or mixed dementia $(\mathrm{HR}=3.66$ (95\% CI 1.29 to 10.41$), p=0.015)$, and no significant association was noted with Alzheimer's disease ( $\mathrm{HR}=1.55$ (95\% CI 0.91 to 2.61), $\mathrm{p}=0.10)$. Several important aspects of this study need to be carefully considered while interpreting findings: (1) patients on urate-lowering therapies (ULTs) were excluded; (2) there was no significant association between sUA levels and MRI markers of cerebrovascular disease or hippocampal volume; and (3) the association between sUA and vascular or mixed dementia was no longer significant, when adjusted for interim

${ }^{1}$ Birmingham VA Medical Center, Birmingham, Alabama, USA

${ }^{2}$ Department of Medicine at the School of Medicine, University of Alabama at Birmingham, Birmingham, Alabama, USA

${ }^{3}$ Division of Epidemiology at the School of Public Health, University of Alabama at Birmingham, Birmingham, Alabama, USA

Correspondence to Dr Jasvinder A Singh, University of Alabama, Faculty Office Tower 805B, 510 20th Street S, Birmingham, AL 35294, USA;

jasvinder.md@gmail.com strokes. The authors carefully noted that these findings were not generalisable to hyperuricaemia or gout cohorts or to those younger than 65 years.

When one examines other studies in this area, the evidence is contradictory. Some studies showed that hyperuricaemia was associated with a lower risk of dementia, ${ }^{2-4}$ while other studies showed an opposite effect. ${ }^{5-10}$ A major limitation is that most of these studies providing the evidence were cross-sectional.

Two recently published systematic reviews carefully examined these data and provide a more comprehensive synthesis of the evidence. The first systematic review assessed whether sUA was associated with cognitive impairment and dementia. ${ }^{11}$ Across 31 studies, using mostly case-control data, sUA was lower in cases of dementia compared with non-dementia controls with a standardised mean difference (SMD) of -0.33 (95 \%CI not provided; $\mathrm{p}<0.001) .{ }^{11}$ In contrast, adjusted logistic regression analysis across five studies suggested no association with increasing sUA (per mg/dL increase) with dementia, with an OR of 1.18 (95\% CI 0.96 to $1.46, \mathrm{p}=0.12)$. $^{11}$ There was no correlation between the sUA level and the scores on MMSE $(r=-0.08$, $\mathrm{p}=0.27$ ), except in patients with Parkinson's-disease-related dementia $(r=0.155$, $\mathrm{p}=0.003) .{ }^{11}$ Major limitations were clinical heterogeneity between studies, the risk of bias in studies including publication bias and a small sample size, and a cross-sectional design for most studies. ${ }^{11}$ The systematic review concluded that the relationship between sUA and dementia/ cognitive impairment was not consistent across all dementia groups.

Another systematic review assessed the association of sUA with Alzheimer's dementia. $^{12}$ Based on 11 case-control studies including 2,708 participants, the sUA levels were not significantly different between patients with Alzheimer's dementia and healthy controls, and the standardized mean difference (SMD; same as the effect size) for sUA was -0.50 (95\% CI -1.23 to 0.22 ), not statistically significant. ${ }^{12}$ Therefore, based on these systematic reviews, there is no convincing evidence to date that higher sUA levels are associated with a lower risk of dementia, except possibly in Parkinson's-disease-related dementia.

The current cohort study draws our attention to the association of the sUA level (hyperuricaemia) with the risk of dementia in the elderly using a population-based sample of the French elderly. ${ }^{1}$ The current study reported an association opposite to what has been a past concern, by showing a significant association of the highest baseline sUA quartile with a 1.8-times higher risk of dementia with up to 12-year follow-up. The study showed that the association of higher sUA level was stronger with vascular or mixed dementia compared with Alzheimer's disease, hinting at different pathogenic mechanisms for these types of dementia as it relates to sUA levels. ${ }^{1}$ The lack of association of sUA levels and MRI markers of cerebrovascular disease is an equally interesting negative finding. This negative finding might be related to a small number of incident cases despite a large cohort sample size and/or low sensitivity of this MRI marker for early/incident dementia. In general, a key challenge to any study of dementia or associated risk factors is its long asymptomatic period and a gradual onset in most cases. These challenges can be addressed by the development of more accurate biomarkers of early dementia, an active area of research that holds promise for the future. ${ }^{13-15}$ Thus, this study adds significantly to the current knowledge base that contains few prospective cohort studies.

This study, ${ }^{1}$ like any well-done study, raises several important questions that future studies should attempt to address: (1) What impact would a change in sUA over time have on the risk of dementia in the elderly? (2) Would the effect be similar in somewhat younger patient populations, that is, those younger than 65 years? (3) Do these risks vary by the presence of cardiovascular or cerebrovascular disease? These are a few questions that this study raises, which can guide the planning of well-designed studies investigating these relationships in the future.

\section{ARE THERE IMPLICATIONS FOR HYPERURICAEMIA AND DEMENTIA RISK IN PATIENTS WITH GOUT?}

A recent treatment guideline extrapolated the concern related to low sUA and the risk of dementia to the treatment for gout, ${ }^{16}$ despite a relative lack of evidence 
from patients with gout. The 2016 European League Against Rheumatism (EULAR) gout treatment guideline stated that for patients with gout being treated with ULT 'sUA level $<3 \mathrm{mg} / \mathrm{dL}$ is not recommended in the long term', ${ }^{16}$ as part of one of the recommendations. They cited few, but not all the studies included in the systematic reviews, ${ }^{11} 12$ and the evidence cited was not from patients with gout. Therefore, the observational evidence used $^{16}$ was low-quality evidence due to serious indirectness. The discrepancy in studies included by the EULAR task force and these systematic reviews may be due to the differences in the inclusion/exclusion criteria. A cautionary EULAR recommendation in the absence of high-quality evidence may appear clinically justified to many but may be viewed by others as needing more evidence before implementation. Lowering of sUA $<3 \mathrm{mg}$ / $\mathrm{dL}$ in patients with gout is not a common occurrence in the clinical practice, and therefore, clinical and research evidence related to it is very limited to none. This recommendation helps to draw more attention to this interesting clinical area, by generating a healthy debate. ${ }^{17}$ The data available to date, including the two systematic reviews discussed above, ${ }^{11} 12$ provide reassurance that the serum urate lowering that is typically achieved with oral ULT is unlikely to contribute to development of dementia (and could have potential benefits).

In the field of gout, there are several unanswered questions about sUA and the risk of dementia and the effect of ULTs on the risk of dementia. Currently, we do not know: (1) if a threshold for sUA exists in gout that is associated with a higher or lower risk of dementia; and (2) whether such lowering is safe for short term, but not long term, that is, is there is a time threshold to sUA lowering? Longitudinal observational studies of an adequate sample of patients with gout as well as observational and randomised studies of ULT in patients with gout can shed some light on these issues. Such studies are now needed to clarify the role of sUA in dementia risk in patients with gout.

\section{WHAT IS NEXT?}

The current study challenged the view that high sUA is protective against the risk of dementia and showed that high sUA was a risk factor for dementia in the elderly general population. More high-quality longitudinal studies like this study assessing the association between sUA and dementia (and early biomarkers) are needed. There is a need for reproduction of these relationships across various cohorts and an examination of the correlation of sUA level with various imaging and functional assessments for cognition and dementia. New knowledge in this area could open a new line/s of investigation for prevention and treatment of dementia.

Contributors JAS designed the editorial, wrote the first draft, made revisions to the manuscript and read and approved the final manuscript.

Funding JAS is supported by the resources and the use of facilities at the VA Medical Center at Birmingham, Alabama, USA.

Competing interests JAS has received research grants from Takeda and Savient and consultant fees from Savient, Takeda, Regeneron, Merz, Iroko, Bioiberica, Crealta/Horizon and Allergan pharmaceuticals, WebMD, UBM LLC and the American College of Rheumatology. JAS serves as the principal investigator for an investigator-initiated study funded by Horizon pharmaceuticals through a grant to DINORA, Inc., a 501 (c)(3) entity. JAS is a member of the executive of OMERACT, an organisation that develops outcome measures in rheumatology and receives arms-length funding from 36 companies; a member of the American College of Rheumatology's (ACR) Annual Meeting Planning Committee; Chair of the ACR Meet-the-Professor, Workshop and Study Group Subcommittee; and a member of the Veterans Affairs Rheumatology Field Advisory Committee. JAS is the editor and the director of the UAB Cochrane Musculoskeletal Group Satellite Center on Network Meta-analysis.

Provenance and peer review Commissioned; externally peer reviewed.

Data sharing statement We used data published in the public domain to write this editorial. These data are available to others through the world wide web and/or local libraries.

(C) Article author(s) (or their employer(s) unless otherwise stated in the text of the article) 2018. All rights reserved. No commercial use is permitted unless otherwise expressly granted.

\section{Check for updates}

To cite Singh JA. Ann Rheum Dis 2018;77:317-318.

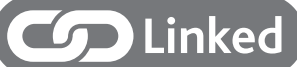

- http://dx.doi.org/10.1136/annrheumdis-2016210767
Ann Rheum Dis 2018;77:317-318.

doi:10.1136/annrheumdis-2017-211975

\section{REFERENCES}

1 Latourte A, Soumaré A, Bardin T, et al. Uric acid and incident dementia over 12 years of follow-up: a population-based cohort study. Ann Rheum Dis 2018;77:328-35.

2 Hong JY, Lan TY, Tang GJ, et al. Gout and the risk of dementia: a nationwide population-based cohort study. Arthritis Res Ther 2015;17:139.

3 Lu N, Dubreuil M, Zhang Y, et al. Gout and the risk of Alzheimer's disease: a population-based, BMI-matched cohort study. Ann Rheum Dis 2016;75

4 Euser SM, Hofman A, Westendorp RG, et al. Serum uric acid and cognitive function and dementia. Brain 2009;132:377-82.

5 Afsar B, Elsurer R, Covic A, et al. Relationship between uric acid and subtle cognitive dysfunction in chronic kidney disease. Am J Nephrol 2011;34:49-54.

6 Cicero AF, Desideri G, Grossi G, et al. Serum uric acid and impaired cognitive function in a cohort of healthy young elderly: data from the Brisighella Study. Intern Emerg Med 2015;10:25-31.

7 Perna L, Mons U, Schöttker B, et al. Association of cognitive function and serum uric acid: are cardiovascular diseases a mediator among women? Exp Gerontol 2016;81:37-41.

8 Verhaaren BF, Vernooij MW, Dehghan A, et al. The relation of uric acid to brain atrophy and cognition: the Rotterdam Scan Study. Neuroepidemiology 2013:41:29-34

9 Beydoun MA, Canas JA, Dore GA, et al. Serum uric acid and its association with longitudinal cognitive change among urban adults. J Alzheimers Dis 2016;52:1415-30.

10 Ruggiero C, Cherubini A, Lauretani F, et al. Uric acid and dementia in community-dwelling older persons. Dement Geriatr Cogn Disord 2009;27:382-9.

11 Khan AA, Quinn TJ, Hewitt J, et al. Serum uric acid level and association with cognitive impairment and dementia: systematic review and meta-analysis. Age 2016;38:16.

12 Chen X, Guo X, Huang R, et al. Serum uric acid levels in patients with Alzheimer's disease: a meta-analysis. PLoS One 2014;9:e94084.

13 Lawrence E, Vegvari C, Ower A, et al. A Systematic review of longitudinal studies which measure alzheimer's disease biomarkers. J Alzheimers Dis 2017;59:1359-79.

14 Frisoni GB, Boccardi M, Barkhof F, et al. Strategic roadmap for an early diagnosis of Alzheimer's disease based on biomarkers. Lancet Neurol 2017;16:661-76.

15 Humpel C. Identifying and validating biomarkers for Alzheimer's disease. Trends Biotechnol 2011;29:26-32.

16 Richette P, Doherty M, Pascual E, et al. 2016 updated EULAR evidence-based recommendations for the management of gout. Ann Rheum Dis 2017;76:29-42.

17 Singh JA, Edwards NL. EULAR gout treatment guidelines by Richette et al.: uric acid and neurocognition. Ann Rheum Dis 2017:annrheumdis-2017-211418. 